\title{
Prevalent osteoporotic vertebral fractures more likely involve the upper endplate than the lower endplate and even more so in males
}

\author{
Nazmi Che-Nordin $^{1 \#}$, Min Deng ${ }^{1 \#}$, James F. Griffith ${ }^{1}$, Jason C. S. Leung ${ }^{2}$, Anthony W. L. Kwok ${ }^{2}$, Yue-Qi \\ Zhu $^{3}$, Richard H. Y. So ${ }^{4}$, Timothy C. Y. Kwok ${ }^{2,5}$, Ping Chung Leung ${ }^{2}$, Yì Xiáng J. Wáng ${ }^{1}$ \\ ${ }^{1}$ Department of Imaging and Interventional Radiology, ${ }^{2} \mathrm{JC}$ Centre for Osteoporosis Care and Control, Faculty of Medicine, The Chinese University \\ of Hong Kong, Prince of Wales Hospital, Shatin, Hong Kong, China; ${ }^{3}$ Department of Diagnostic and Interventional Radiology, Shanghai Jiaotong \\ University Affiliated Sixth People's Hospital, Shanghai 200233, China; ${ }^{4}$ Department of Industrial Engineering and Decision Analytics, Hong Kong \\ University of Science and Technology, Clearwater Bay, Hong Kong, China; ${ }^{5}$ Department of Medicine and Therapeutics, Faculty of Medicine, The \\ Chinese University of Hong Kong, Prince of Wales Hospital, Shatin, Hong Kong, China \\ Contributions: (I) Conception and design: YX Wáng; (II) Administrative support: JC Leung, AW Kwok; (III) Provision of study materials or patients: \\ AW Kwok, TC Kwok, PC Leung; (IV) Collection and assembly of data: M Deng, N Che-Nordin, JC Leung; (V) Data analysis and interpretation: N \\ Che-Nordin, M Deng, JF Griffith, YQ Zhu, RH So, YX Wáng; (VI) Manuscript writing: All authors; (VII) Final approval of manuscript: All authors. \\ \#These authors contributed equally to this work. \\ Correspondence to: Yì Xiáng J. Wáng, MMed, PhD. Department of Imaging and Interventional Radiology, Faculty of Medicine, The Chinese \\ University of Hong Kong, Prince of Wales Hospital, Shatin, New Territories, Hong Kong, China. Email: yixiang_wang@cuhk.edu.hk.
}

Background: While the importance of identifying osteoporotic vertebral endplate fracture (EPF) is being recognized; the pathophysiological understanding of EPF till now remain insufficient. In this populationbased cross-sectional radiograph study, we aim to investigate the anatomic location characteristics of osteoporotic EPF.

Methods: This study analyzed the anatomical location of osteoporotic EPFs in elderly Chinese population (age $\geq 65$ years). The T4-L4 radiographs of 1,954 elderly Chinese men (mean: 72.3 years) and 1,953 elderly Chinese women (mean: 72.5 years) were evaluated to identify EPF, and vertebral bodies were graded according to Genant's vertebral deformity criteria.

Results: Of the 101,582 endplates analyzed, there were 505 EPFs (males: 27.7\%; females: 72.3\%). Excluding those with both upper endplate and lower endplate involvements, the ratio of upper EPF to lower EPF was 9.63 for males and 4.3 for females $(\mathrm{P}<0.05)$. Thoracolumbar junction, particularly L1 $(26.4 \%$ for males and $24.1 \%$ for females) and followed by T12 (20.7\% for males and $19.7 \%$ for females), had highest prevalence of EPF. With an endplate divided into 5 segments of equal length in the anteroposterior direction and grade $0.5 \& 1$, grade 2 vertebral deformities analyzed, fractures occurred mostly at the middle segment (70.1\% for upper endplates in males and $78.6 \%$ for upper endplates in females), followed by second anterior segment (20.9\% for upper endplates in males and $14.4 \%$ for upper endplates in females). The most anterior and most posterior segments were not primarily involved in EPF.

Conclusions: Osteoporotic EPFs more likely involve the upper endplate rather than lower endplate, with a trend for this effect to be greater in men than in women. These characteristics may help radiographic differential diagnosis for osteoporotic EPF.

Keywords: Epidemiology; radiograph; osteoporosis; osteoporotic fractures; endplate

Submitted Sep 02, 2018. Accepted for publication Oct 12, 2018.

doi: $10.21037 / \mathrm{atm} .2018 .10 .61$

View this article at: http://dx.doi.org/10.21037/atm.2018.10.61 


\section{Introduction}

Osteoporosis is characterized by low bone mass and microarchitectural deterioration, which leads to bone fragility and consequent increase in fracture risk. Vertebral fractures (VFs) are the most common osteoporotic fracture. A VF, after minor trauma, is a hallmark of osteoporosis. Prevalent VFs increase the risk of future vertebral and non-vertebral osteoporotic fracture independent of bone mineral density (BMD). VFs are associated with poor life quality, impaired bending and rising, difficulties in the activities of daily living, frailty, higher risk of hospitalization, and higher mortality $(1,2)$. Appropriate management of osteoporosis can reduce future fracture risk, it is important to identify and report VFs accurately and clearly, so that appropriate investigation and treatment can be initiated (3).

Currently the radiographic criteria for osteoporotic $\mathrm{VF}$ and its grading remain debated (3-14). On spine radiograph, the semi-quantitative (SQ) criteria proposed by Genant et al. is commonly used for identifying VF from vertebrae T4 to L4 (15). Genant et al. described the importance of loss of end-plate integrity as a characteristic of $\mathrm{VF}$ but did not make diagnosis contingent on this sign $(12,15)$. The algorithm-based qualitative (ABQ) approach, initially described by Jiang et al., assumes that the endplate (EP) is always deformed in VFs and is a $100 \%$ sensitive in cases of VF, while vertebral height reduction is not an indispensable finding of VF $(16,17)$. Controversies remain, such as whether osteoporotic VF can actually occur without radiographically identifiable endplate fracture (EPF); and whether without endplate disruption, osteoporotic VF fracture only deform the anterior vertebral cortex which have commonly been termed as buckling, dented, swollen and projecting types of fracture $(3,5,7,14,15)$. The current evidences favor the approaches incorporating EPF identification for VF assessment $(3,4,7,13,17)$. For example, Lentle et al. showed ABQ grade $1 \mathrm{VF}$ was associated with higher risk of $\mathrm{VFs}$ as well as nonvertebral major osteoporotic fracture, while grade $1 \mathrm{SQ}$-vertebral deformity (VD) was not associated with higher non-VF (7). We showed subjects with grade 1 SQ-VD had a similar BMD compared with subjects without fracture, while subjects with grade $1 \mathrm{ABQ}$ VF had lower BMD (13). Thus, $\mathrm{EPF}$ can be a distinct sign of $\mathrm{VF}$ and should be recognized, though the absence of radiographically identifiable EPF does not necessarily exclude the existence of an osteoporotic VF $(3,5,14,18-21)$.

While the importance of identifying vertebral endplate/ cortex fracture has been recognized, evaluation of osteoporotic endplate/cortex fracture can be difficult and requires training and experience $(5,14)$. The pathophysiological understanding of osteoporotic EPF till now remain insufficient. Osteoporotic Fractures in Men (MrOS) (Hong Kong) and Osteoporotic Fractures in Women (MsOS) (Hong Kong) represent the first largescale prospective cohort studies ever conducted on bone health in Asian men and women (22). Interestingly, the results demonstrated that the age-specific osteoporotic VF prevalence of Chinese women is very similar to Japanese and Korean women as well as Latin American women, suggesting the difference in VF epidemiology among different ethnic groups might be smaller than initially thought (22). Using the baseline radiograph data, the purpose of this study is to investigate the anatomic location characteristics of EPF, including upper (cranial) endplate fracture (uEPF) vs. lower (caudal) endplate fracture (lEPF), fracture location in anterior-posterior (AP) direction of endplate, and distribution among different thoracolumbar vertebral levels as well as gender differences.

\section{Methods}

For MrOS (Hong Kong) and MsOS (Hong Kong) baseline studies, 2,000 Chinese men and 2,000 Chinese women aged 65 or above were recruited from the local communities by advertisements placed in housing estates and community centers for elderly people to take part in a prospective study from August 2001 to March 2003, to determine the relationship between anthropometric, lifestyle, medical, and other factors with BMD at the hip and spine. The recruitment plan was designed so that the participants would represent the general elderly population in age and gender proportion. The project was designed primarily to examine the BMD of older Chinese adults prospectively for 4 years. All subjects were community dwelling, able to walk without assistance, without bilateral hip replacement, and had the potential to survive the duration of the primary study as judged by their preexisting medical status. The study protocol was approved by the Chinese University of Hong Kong Ethics Committee, with written informed consent obtained for all participants.

Left lateral thoracic and lumbar spine radiographs were obtained by adjusting the exposure parameters according to participants' body weight and height. Data from the baseline evaluation were analyzed in the current study, and the reading procedure has been described previously 
$(5,10,14)$. Hard copy film was used for analysis primarily, aided with digitalized format using Philips DICOMViewer (Philips Healthcare, Best, The Netherlands). Vertebrae T4-L4 were evaluated. Visualizing T1-T3 is often limited due to overlying of the shoulders and L5 due to overlying pelvis. Osteoporotic VF above T4 is also very rare. There is no minimum threshold for reduction in vertebral height for a prevalent EPF (16). As described previously, in additional to qualitative radiological assessment $(12,15,23)$, quantitative measurement was performed, grade 0.5 \& 1 refers to an involved vertebra had deformity but with $<25 \%$ height loss; grade 2 refers to an involved vertebra had deformity and with $25-40 \%$ height loss; and grade 3 refers to an involved vertebra had deformity and with $>40 \%$ height loss. The common developmental wedge deformities and the common mild endplate bowing of the lower lumbar vertebrae were recognized. Non-fractural changes of the vertebrae shape, such as developmental short vertebral height, Cupid's bow deformity, Scheuermann's disease, and Schmorl's nodes, and degenerative remodeling, were excluded $(5,14)$. In our previous study, without quantitative measurement we found the kappa for inter-reader agreement of 0.75 for $\mathrm{VD}$ reading (22). The main discord for inter-reader disagreement related to the borderline cases, such as a perceived reduction in vertebral body height of approximate $25 \%$ could be categorized as grade 1 or grade 2 VF. Since quantitative measurement was adopted, we expected a better kappa value would have been achieved. Our intra-reader agreement kappa was tested to 0.78 for the ECF method, which was similar to the result of Ferrar et al. $(10,17)$. Two readers, both experienced radiologists, assessed the radiographs simultaneously according to Genant's SQ and ECF criteria, and consensus was reached (10). During the current analysis of EPF location, the reading of EPF was additionally double-checked by a trained radiographer, one of the initial radiologist reader and another radiologist experienced in VF reading were available for consultation and discussion during the checking.

For the assessment of the AP location of EPF, the magnification of the endplate was adjusted on the computer screen and compared with a transparent ruler so that a magnified endplate measured 5 inches, and thus divided into 5 segments (from anterior to posterior: a1, a2, m, p2, $\mathrm{p} 1$ ), and then the lowest point of an EPF was recorded. AP location of EPF was assessed for grade $0.5 / 1$ and grade 2 vertebrae, as grade 3 deformities have a 'collapse' of vertebra and difficult to evaluate for this purpose. Examples of a uEPF, a IEPF, and 5-segment partition of endplate are shown in Figure 1.

Forty-six spine radiographs (2.3\%) for males and 47 spine radiographs $(2.35 \%)$ for females (out of 2,000 for each group) were adjudged to be of suboptimal film quality for EPF assessment, leaving 1,954 male subjects (mean age: 72.3 years, range, 65-92 years) and 1,953 females (mean age: 72.5 years, range, 65-98 years) for final analysis of 101,582 endplates. There was no difference in age between the 2 sexes $(\mathrm{P}=0.417)$. Suboptimal film quality included scoliosis subjects and films with insufficient $\mathrm{X}$-ray exposure. None of these subjects' spines were diagnosed as having pathological fractures or diseases other than degenerative or osteoporotic change.

Statistical analysis was performed using SPSS version 24.0 (SPSS Inc., Illinois, US) with, and an $\alpha$ level of $5 \%$ was used as the level of significance. Prevalence of VFs with both uEP and IEP was compared among three VD groups by using Chi square test for trend. Prevalence was compared between males and females by using Chi square test of independence.

\section{Results}

Of the 101,582 EP analyzed, there were in total $505 \mathrm{EPFs}$, with $27.7 \%$ in males and $72.3 \%$ in females. The EPF results for males are shown in Table 1 and Figure S1. In total there were $140 \mathrm{EPFs}$, involving $77 \mathrm{uEP}$ and 8 IEP, and 55 (39.3\%) VFs had both uEP and IEP involvement. Excluding those with both $\mathrm{uEP}$ and IEP involvement, the uEPF/lEPF ratio was 9.6 (95\% CI: 5.4-30.3) for males. EPF involved lumbar vertebra more often than thoracic vertebra, most often at L1 vertebra (26.4\%), followed by vertebra T12 (20.7\%). The highest EPF prevalence at mid thoracic spine were at the vertebral levels of T6/7/8/9 $(2.9 \%, 4.3 \%, 2.1 \%$, and $4.3 \%$ respectively).

The EPF results for females are shown in Table 2 and Figure S2. In total there were $365 \mathrm{EPFs}$, involving 155 uEPs and 36 lEPs, and 174 (47.7\%) VFs had both uEP and IEP involvement. Excluding those with both uEP and IEP involvement, the ratio of uEPF/lEPF was 4.3 (95\% CI: 3.16.5, compared with the ratio of 9.6 for men, $\mathrm{P}=0.048)$. EPF involved lumbar vertebra more often than thoracic vertebra, most often at L1 (24.1\%), followed by T12 (19.7\%). The highest EPF prevalence at mid thoracic spine were at the vertebral T8 (5.8\%).

For both males and females, grade 3 VDs are more likely to have VF with both uEP and IEP involvement (Tables 1,3, $\mathrm{P}<0.001$ for trend). Females were more likely to have VFs 

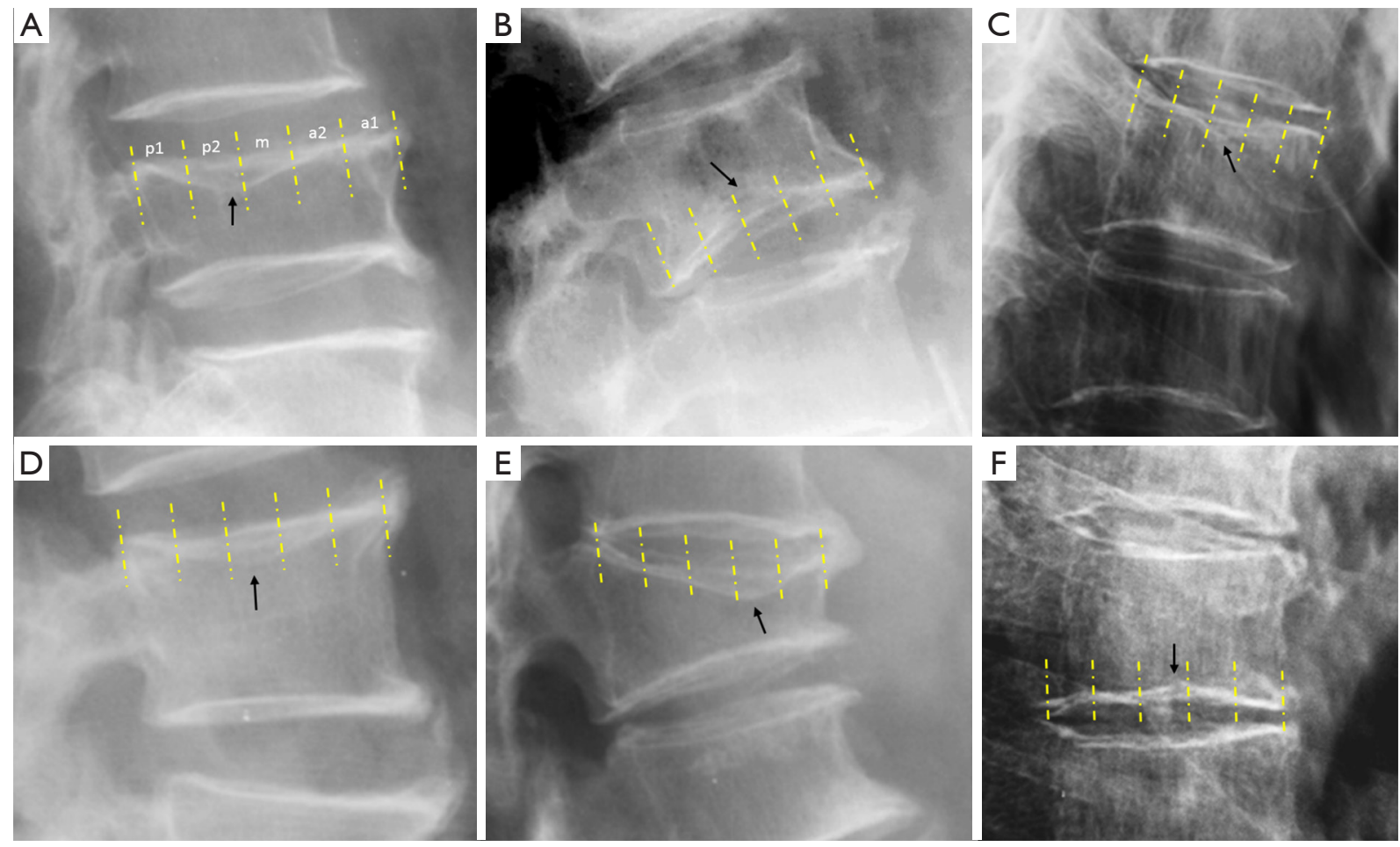

Figure 1 Six vertebrae with endplate fracture. Arrows point to the lowest point of endplate depression, with uEP-p2 for (A), lEP-m for (B), $\mathrm{uEP}-\mathrm{m}$ for (C), uEP-m for (D), uEP-a2 for (E), and lEP-m for (F). Note the vertebrae in (C) and (D) each has an upper endplate fracture (uEPF), and the vertebra in $(\mathrm{F})$ has a lower endplate fracture (lEPF), but all without apparent vertebral body height loss. Yellow lines separate each endplate into 5 segments of equal anteroposterior length.

with both uEP and lEP involvement (47.7\%) than males (39.3\%, $\mathrm{P}=0.0902)$.

For both males and females and both uEP and IEP, in endplate AP direction fractures occurred often at segment-m, followed by segment-a 2 and then by segment-p2 (Table 3). No EPF was primarily observed at segment-a 1 and segment-p1. For EPF at A-P direction, there was no apparent trend of differences observed at different vertebral levels (details see Figure S1,S2).

\section{Discussion}

A vertebral endplate consists of perforated cortical bone with a layer of hyaline cartilage bonded to its disc surface. The cortical bone layer contains a network of small cavities which allow bone marrow to lie adjacent to calcified hyaline cartilage for approximately $10 \%$ of the central endplate area, which is an important route for metabolite transport into the discs. The nutritional demands of the discs result in that vertebral endplates are thin and porous. EPF may cause back pain and can lead to disc degeneration (24-26). It has been known that uEPs are injured more often than IEPs, as uEPs are thinner and less supported by trabecular bone (27-29). Zhao et al. (27) reported that uEPs are thinner than $1 \mathrm{EPs}$ by $14 \%$ and $11 \%$ on average, in mid-sagittal and pedicle slices respectively. The underlying cancellous bone of uEP is less dense than that of the lEP (27). Mechanical experiments on cadaveric spines confirm that uEP is more vulnerable to compressive damage than $\operatorname{lEP}(30,31)$. Trabecular arcades from the pedicles may reinforce the IEPs more than uEPs (32). These structural variations in upper and lower endplates may be related to inherent spinal characteristics rather than aging $(27,29)$. Wang et al. (29) reported that structural asymmetries of vertebral trabeculae were not associated with age or disc degeneration.

In an ex vivo biomechanical testing, Zhao et al. (27) reported compressive loading resulted in uEP damaged in 55 out of 57 specimens and the lEP damaged in only two (2/57; $48 \%$ females, age: $81 \pm 9$ years for 17 females and $76 \pm 10$ years for 18 males). In a patient study, Trout et al. (33) reported that prevalent VFs in an osteoporotic population occur more likely at uEP. They analyzed 84 patients 
Table 1 Distribution of osteoporotic endplate fracture (EPF) in male subjects

\begin{tabular}{|c|c|c|c|c|c|c|c|c|c|c|c|c|}
\hline \multirow{2}{*}{$\begin{array}{l}\text { Vertebral } \\
\text { level }\end{array}$} & \multicolumn{3}{|c|}{ Grade 0.5/1 VD } & \multicolumn{3}{|c|}{ Grade 2 VD } & \multicolumn{3}{|c|}{ Grade 3 VD } & \multirow{2}{*}{ Total } & \multirow{2}{*}{$\%$} & \multirow{2}{*}{$95 \% \mathrm{Cl}$} \\
\hline & uEP & IEP & Both & uEP & IEP & Both & uEP & IEP & Both & & & \\
\hline T4 & 0 & 0 & 0 & 0 & 0 & 0 & 0 & 0 & 1 & 1 & 0.7 & $0-2.1$ \\
\hline T5 & 1 & 0 & 0 & 1 & 0 & 0 & 0 & 0 & 1 & 3 & 2.1 & $0-4.5$ \\
\hline T6 & 0 & 0 & 0 & 1 & 0 & 1 & 1 & 0 & 1 & 4 & 2.9 & $0.1-5.6$ \\
\hline $\mathrm{T} 7$ & 2 & 0 & 0 & 2 & 0 & 1 & 0 & 0 & 1 & 6 & 4.3 & $0.9-7.6$ \\
\hline T8 & 1 & 0 & 0 & 0 & 1 & 0 & 0 & 0 & 1 & 3 & 2.1 & $0-4.5$ \\
\hline T9 & 2 & 0 & 0 & 2 & 0 & 0 & 0 & 0 & 2 & 6 & 4.3 & $0.9-7.6$ \\
\hline T10 & 0 & 0 & 0 & 0 & 0 & 0 & 0 & 0 & 1 & 1 & 0.7 & $0-2.1$ \\
\hline T11 & 2 & 1 & 0 & 3 & 0 & 1 & 1 & 0 & 2 & 10 & 7.1 & $2.9-11.4$ \\
\hline T12 & 1 & 0 & 0 & 6 & 3 & 0 & 6 & 0 & 13 & 29 & 20.7 & $14.0-27.4$ \\
\hline L1 & 0 & 0 & 0 & 9 & 2 & 3 & 13 & 0 & 10 & 37 & 26.4 & $19.1-33.7$ \\
\hline L2 & 2 & 1 & 0 & 6 & 0 & 2 & 1 & 0 & 5 & 17 & 12.1 & $6.7-17.6$ \\
\hline L3 & 2 & 0 & 0 & 5 & 0 & 2 & 1 & 0 & 2 & 12 & 8.6 & 3.9-13.2 \\
\hline L4 & 1 & 0 & 1 & 3 & 0 & 2 & 2 & 0 & 2 & 11 & 7.9 & $3.4-12.3$ \\
\hline Total & 14 & 2 & 1 & 38 & 6 & 12 & 25 & 0 & 42 & 140 & & \\
\hline $\mathrm{u} / \mathrm{l}$ ratio & & & & & & & & & & & & \\
\hline$\%$ both & & & $5.9 \%^{*}$ & & & $21.4 \% *$ & & & $62.7 \% *$ & & & \\
\hline
\end{tabular}

*, $\mathrm{P}<0.001$. UEP, upper endplate; IEP, lower endplate; both, involvement of both upper and lower endplates; \%, total EFPs at each vertebral level divided by total EPFs of this male database, i.e., $n=140$. u/l ratio, ratio of uEPF/IEPF at each vertebral deformity (VD) grade. $\%$ both, percentage of EPF involving both UEP and IEP at each VD grade.

(median age: 72.5 years, $67.4 \%$ females), with 312 prevalent osteoporotic VFs at baseline, 57\% involved uEPs, 11\% involved $\mathrm{IEPs}$ ( $\mathrm{uEPF} / \mathrm{lEPF}$ ratio $=5.2$ ), and $32 \%$ involved both upper and lower endplates. On the other hand, they noted that incident VFs that occur in vertebrae immediately cephalad to a cemented vertebra with vertebroplasty localized disproportionally to $1 \mathrm{EP}$ ( $30 \% \mathrm{uEPs}, 57 \% \mathrm{lEPs})$. Our results agree with the Trout et al.'s on uEPF/lEPF ratio of prevalent osteoporotic VFs (our data: 5.1 for combined males females results); however, we had more VFs involved both uEP and IEP (44.3\% for combined males females results). It is more likely that VFs involved both $\mathrm{uEP}$ and IEP represent a progression of either $\mathrm{uEPF}$ or IEPF. It is understandable that, as shown in our results, as the VF severity progresses from grade 1 to grade 3, the proportion of VFs involving both $\mathrm{uEP}$ and IEP increases. One interesting finding from our study is that, the lEP is distinctly more less likely to fracture in males compared with females (uEPF/IEPF ratio $=9.63$ for males, and $=4.3$ for females). Males and females spine have different VF characteristics. Compared with females and in relatively term, endplate in males (and particularly lEP in males) may be stronger and more resilient to compressive force that osteoporotic vertebral compromise more likely appears as deformity and height loss rather than as endplate or cortex fracture $(10,13)$.

It is known that there is in-plane regional variation in thickness of EP, being greater adjacent to the annulus than the nucleus (34). In addition, the central endplate has a greater concentration of marrow contact channels in the region which is known to be most porous (35), as nutritional demands are greatest in the disc nucleus. Trabecular density is lower in anterior and middle regions compared to posterior regions (27). Thickening of lEPs relative to uEPs is most marked in posterior regions of pedicles, with IEP being thickened and strengthened by trabecular arcades from the pedicles (32). The mechanical significance of morphological differences in the vertebrae 
Table 2 Distribution of osteoporotic endplate fracture (EPF) in female subjects

\begin{tabular}{|c|c|c|c|c|c|c|c|c|c|c|c|c|}
\hline $\begin{array}{l}\text { Vertebral } \\
\text { level }\end{array}$ & \multicolumn{3}{|c|}{ Grade 0.5/1 VD } & \multicolumn{3}{|c|}{ Grade 2 VD } & \multicolumn{3}{|c|}{ Grade 3 VD } & Total & $\%$ & $95 \% \mathrm{Cl}$ \\
\hline T4 & 0 & 0 & 0 & 0 & 0 & 0 & 0 & 0 & 2 & 2 & 0.5 & $0-1.3$ \\
\hline T5 & 1 & 1 & 0 & 1 & 0 & 2 & 0 & 0 & 3 & 8 & 2.2 & $0.7-3.7$ \\
\hline T6 & 2 & 0 & 0 & 0 & 0 & 4 & 1 & 0 & 2 & 9 & 2.5 & $0.9-4.1$ \\
\hline T8 & 1 & 0 & 0 & 3 & 1 & 6 & 2 & 0 & 8 & 21 & 5.8 & $3.4-8.1$ \\
\hline T9 & 0 & 1 & 0 & 1 & 1 & 4 & 0 & 1 & 2 & 10 & 2.7 & $1.1-4.4$ \\
\hline T10 & 0 & 2 & 1 & 3 & 3 & 1 & 1 & 2 & 4 & 17 & 4.7 & $2.5-6.8$ \\
\hline T11 & 4 & 1 & 0 & 6 & 1 & 3 & 0 & 0 & 8 & 23 & 6.3 & $3.8-8.8$ \\
\hline L2 & 10 & 0 & 0 & 15 & 3 & 6 & 3 & 0 & 11 & 48 & 13.1 & $9.7-16.6$ \\
\hline L3 & 5 & 3 & 1 & 11 & 4 & 7 & 1 & 0 & 5 & 37 & 10.1 & $7.0-13.2$ \\
\hline L4 & 2 & 0 & 1 & 6 & 0 & 5 & 0 & 0 & 3 & 17 & 4.7 & $2.5-6.8$ \\
\hline total & 43 & 12 & 4 & 84 & 17 & 57 & 28 & 7 & 113 & 365 & & \\
\hline $\mathrm{u} / \mathrm{l}$ ratio & \multicolumn{3}{|c|}{3.6} & \multicolumn{2}{|c|}{5.0} & & \multicolumn{2}{|c|}{4} & & & & \\
\hline$\%$ both & & & $6.8 \%{ }^{*}$ & & & $36.1 \%$ * & & & $76.4 \%$ * & & & \\
\hline
\end{tabular}

uEP, upper endplate; IEP, lower endplate; both, involvement of both upper and lower endplates; \%, total EFPs at each vertebral level divided by total EPFs of this female database, i.e., $n=365$. u/l ratio, ratio of uEPF/IEPF at each vertebral deformity (VD) grade. \% both, percentage of EPF involving both UEP and IEP at each VD grade. ${ }^{*} \mathrm{P}<0.001$

Table 3 In plane distribution of osteoporotic endplate fracture (EPF, grade 3 deformities were not included in analysis)

\begin{tabular}{|c|c|c|c|c|c|c|c|c|c|c|}
\hline $\begin{array}{l}\text { Male or female } \\
\text { endplate fracture }\end{array}$ & \multicolumn{5}{|c|}{ uEP } & \multicolumn{5}{|c|}{ IEP } \\
\hline Males EPF No. & 0 & 6 & 47 & 14 & 0 & 0 & 1 & 12 & 5 & 0 \\
\hline Males \% EPF & 0 & 9.0 & 70.1 & 20.9 & 0 & 0 & 5.6 & 66.7 & 27.8 & 0 \\
\hline Females EPF No. & 0 & 13 & 147 & 27 & 0 & 0 & 2 & 64 & 20 & 0 \\
\hline
\end{tabular}

uEP, upper endplate; IEP, lower endplate; EPF No., EPF number at each EP segment. No EPF is primarily seen at P1 and a1 segments. \% EPF, percentage of EPF number at each EP segment with UEP and IEP calculated separately. See Figure 1 for the definition of each segment. Males uEP a2 vs. P2, $\mathrm{p}=0.074$, IEP a2 vs. $\mathrm{p} 2, \mathrm{P}=0.102$; females, uEP a2 vs. $\mathrm{p} 2, \mathrm{P}=0.027, \mathrm{IEP}$ a2 vs. $\mathrm{p} 2, \mathrm{P}<0.001$.

is also supported by Grant et al. who used an indentation technique to demonstrate that lEPs are stronger than uEPs $(31,36)$. These anatomical observations explain why, for most EPFs, the lowest point, i.e., the most fractured point or maybe the fracture initiation point, occur in the central segment (m) of the EP, and then followed by segment-a2 of the EP (Figure 1). This study did not show EPF initially occurred at segment-a1 and segment-p1, i.e., EPF did not occur at the most anterior and most posterior $1 / 5$ segments of EP. However, it remains to be further confirmed by even larger studies that actually EPF would not initiate at these regions.

This study shows, for both males and females, thoracolumbar junction had the highest prevalence of 
osteoporotic EPF, and notably L2 and L3 vertebrae were also frequently involved. It has been noted that lumbar endplates in the upper region are thinner than in the lower region (28). In addition to biomechanical stress at thoracolumbar junction, this helps to additionally explain why EPFs/VFs are more common in the upper lumbar region than in the lower region. The distribution of osteoporotic EPF at different vertebral levels is in line with the distribution of osteoporotic VD according to SQ criteria (10). IEPs in lower lumbar region may be relatively thickened as a result of the nutritional demands of the larger discs caudally. Edwards et al. (35) noted that endplates are thicker at lower lumbar levels. Yang et al. (28) also reported that vertebral trabecular parameters (such as higher BMD and trabecular bone volume, greater trabecular number, greater connectivity density, and less trabecular separation) cranial to the disc were greater than those caudally in the upper but not in the lower lumbar region. However, our results showed, both for males and females, $\mathrm{uEP}$ were more likely to fracture than IEP at least till L4 vertebra level.

This study has a few limitations. EPF evaluation was based on radiograph, while due to its projectional nature radiographic evaluation may miss EPF evident on microscopy or on high resolution cross-sectional CT scans (5). High resolution CT study is not routinely indicated for osteoporotic spine fracture assessment. While we have been working on EPF for a number of years, and all EPFs in this study have been triple-checked, radiographic evaluation of $\mathrm{EPF}$ is subjective and there is no golden standard. Another possibility is that a few osteoporotic-looking EPFs might be initially traumatic due to an unreported (forgotten) old injury. However, even such possibility may exist, the cases would be rare in this carefully executed epidemiological study with follow-up data available for potential validation [some data on year 4 follow-up have been analyzed and parts published (37), and some subjects had year-13 follow-up completed and parts already analyzed]. Fragility fractures are fractures that result from mechanical forces that would not ordinarily result in fracture, i.e., low-level (or 'low energy') trauma. The World Health Organization quantifies this as forces equivalent to a fall from a standing height or less. While the type and direction of this external low-energy force may affect the location and shape of EPF, this could not be explored in the current study and thus constitute another limitation. However, we expect these limitations would not affect the overall conclusion of this study.

In conclusion, this study demonstrates osteoporotic
EPFs more likely involve the uEP than the lEP, with a trend for this effect to be greater in men than in women. EPFs locate mostly at middle $1 / 5$ segment $(\mathrm{m})$ and the second anterior(a)/second posterior $1 / 5$ segments (p2) of endplate, and EPFs mostly occur at T11-L3 vertebrae. Currently plain radiograph is the recommended imaging technique for osteoporotic VF assessment $(2,12)$. The knowledge derived from this study may contribute to osteoporotic VF clinical management $(2,9,25,38-41)$. Radiologists may pay even closer attention to the most vulnerable anatomical locations for screening EPF. These knowledges can also help differential diagnosis. For example, as Trout et al. (33) and Han et al. (42) noted, new incident lower endplate fractures that occur in vertebrae immediately adjacent to a cemented vertebra is more likely associated with vertebroplasty procedure. While Cupid's bow is often seen at IEP of lower lumbar vertebrae $(5,43)$, osteoporotic EPF rarely involves these locations. The location of EPF in AP direction may also help differential diagnosis with Schmorl's nodes in some cases (5). Additionally, we expect the EPF 'rules' characteristic described in this study may also help designing artificial-intelligence enabled automated detection or semi-automated detection for osteoporotic VF.

\section{Acknowledgements}

We thank Dr. Lai-Chang He at Nanchang University, for helps in interpreting spine radiographs.

Funding: This study was partially funded by National Institutes of Health R01 Grant AR049439-01A1 and the Research Grants Council Earmarked Grant CUHK 4101/02M.

\section{Footnote}

Conflicts of Interest: The authors have no conflicts of interest to declare.

Ethical Statement: The study protocol was approved by the Joint Chinese University of Hong Kong - New Territories East Cluster Clinical Research Ethics Committee (No. CRE-2003-102), with written informed consent obtained for all participants.

\section{References}

1. Klotzbuecher CM, Ross PD, Landsman PB, et al. Patients with prior fractures have an increased risk of future 
fractures: a summary of the literature and statistical synthesis. J Bone Miner Res 2000;15:721-39.

2. Kendler DL, Bauer DC, Davison KS, et al. Vertebral Fractures: Clinical Importance and Management. Am J Med 2016;129:221.e1-10.

3. Szulc P. Vertebral Fracture: Diagnostic Difficulties of a Major Medical Problem. J Bone Miner Res 2018;33:553-9.

4. Lentle BC, Oei E, Goltzman D, et al. Vertebral fractures and morphometric deformities. J Bone Miner Res 2018;33:1544-5.

5. Wáng YX, Santiago RF, Deng M, et al. Identifying osteoporotic vertebral endplate and cortex fractures. Quant Imaging Med Surg 2017;7:555-91.

6. Diacinti D, Vitali C, Gussoni G, et al. Misdiagnosis of vertebral fractures on local radiographic readings of the multicentre POINT (Prevalence of Osteoporosis in INTernal medicine) study. Bone 2017;101:230-5.

7. Lentle BC, Berger C, Probyn L, et al. for the CaMos Research Group. Comparative Analysis of the radiology of osteoporotic vertebral fractures in women and men: crosssectional and longitudinal observations from the Canadian Multicentre Osteoporosis Study (CaMos). J Bone Miner Res 2018;33:569-79.

8. Oei L, Koromani F, Breda SJ, et al. Osteoporotic vertebral fracture prevalence varies widely between qualitative and quantitative radiological assessment methods: the Rotterdam Study. J Bone Miner Res 2018;33:560-8.

9. Fink HA, Litwack-Harrison S, Ensrud KE, et al. for Osteoporotic Fractures in Men (MrOS) Study Group. Association of Incident, Clinically Undiagnosed Radiographic Vertebral Fractures With Follow-Up Back Pain Symptoms in Older Men: the Osteoporotic Fractures in Men (MrOS) Study. J Bone Miner Res 2017;32:2263-8.

10. Deng M, Zeng XJ, He LC, et al. Osteoporotic Vertebral Fracture Prevalence in Elderly Chinese Men and Women: A Comparison of Endplate/Cortex Fracture-Based and Morphometrical Deformity-Based Methods. J Clin Densitom 2017. [Epub ahead of print].

11. Oei L, Koromani F, Rivadeneira F, et al. Quantitative imaging methods in osteoporosis. Quant Imaging Med Surg 2016;6:680-98.

12. Schwartz EN, Steinberg D. Detection of vertebral fractures. Curr Osteoporos Rep 2005;3:126-35.

13. Deng M, Kwok TCY, Leung JCS, et al. All osteoporotically deformed vertebrae with $>34 \%$ height loss have radiographically identifiable endplate/cortex fracture. J Orthop Translat 2018;14:63-6.

14. Wáng YX, Deng M, He LC, et al. Osteoporotic vertebral endplate and cortex fractures: a pictorial review. J Orthop Translat 2018;15:35-49.

15. Genant HK, Wu CY, van Kuijk C, et al. Vertebral fracture assessment using a semiquantitative technique. J Bone Miner Res 1993;8:1137-48.

16. Jiang G, Eastell R, Barrington NA, et al. Comparison of methods for the visual identification of prevalent vertebral fracture in osteoporosis. Osteoporos Int 2004;15:887-96.

17. Ferrar L, Jiang G, Clowes JA, et al. Comparison of densitometric and radiographic vertebral fracture assessment using the algorithm-based qualitative (ABQ) method in postmenopausal women at low and high risk of fracture. J Bone Miner Res 2008;23:103-11.

18. Pham T, Azulay-Parrado J, Champsaur P, et al. "Occult" osteoporotic vertebral fractures: vertebral body fractures without radiologic collapse. Spine (Phila Pa 1976) 2005;30:2430-5.

19. Takahara K, Kamimura M, Nakagawa H, et al. Radiographic evaluation of vertebral fractures in osteoporotic patients. J Clin Neurosci 2007;14:122-6.

20. Amling M, Pösl M, Ritzel H, et al. Architecture and distribution of cancellous bone yield vertebral fracture clues. A histomorphometric analysis of the complete spinal column from 40 autopsy specimens. Arch Orthop Trauma Surg. 1996;115:262-9.

21. Antonacci MD, Mody DR, Rutz K, et al. A histologic study of fractured human vertebral bodies. J Spinal Disord Tech 2002;15:118-26.

22. Kwok AW, Gong JS, Wang YX, et al. Prevalence and risk factors of radiographic vertebral fractures in elderly Chinese men and women: results of Mr. OS (Hong Kong) and Ms. OS (Hong Kong) studies. Osteoporos Int 2013;24:877-85.

23. Wáng YX, Che-Nordin N. Informed communication with study subjects of radiographically detected osteoporotic vertebral deformity. Quant Imaging Med Surg 2018;8:876-80.

24. Kerttula LI, Serlo WS, Tervonen OA, et al. Post-traumatic findings of the spine after earlier vertebral fracture in young patients: clinical and MRI study. Spine (Phila Pa 1976) 2000;25:1104-8.

25. Kokkonen SM, Kurunlahti M, Tervonen O, et al. Endplate degeneration observed on magnetic resonance imaging of the lumbar spine: correlation with pain provocation and disc changes observed on computed tomography diskography. Spine (Phila Pa 1976) 2002;27:2274-8.

26. Holm S, Holm AK, Ekstrom L, et al. Experimental disc degeneration due to endplate injury. J Spinal Disord Tech 
2004;17:64-71.

27. Zhao FD, Pollintine P, Hole BD, et al. Vertebral fractures usually affect the cranial endplate because it is thinner and supported by less-dense trabecular bone. Bone 2009;44:372-9.

28. Yang G, Battié MC, Boyd SK, et al. Cranio-caudal asymmetries in trabecular architecture reflect vertebral fracture patterns. Bone 2017;95:102-7.

29. Wang Y, Battie MC, Boyd SK, et al. The osseous endplates in lumbar vertebrae: thickness, bone mineral density and their associations with age and disk degeneration. Bone 2011;48:804-9.

30. Brinckmann P, Biggemann M, Hilweg D. Fatigue fracture of human lumbar vertebrae. Clin Biomech (Bristol, Avon). 1988;3 Suppl 1:i-S23.

31. Grant JP, Oxland TR, Dvorak MF. Mapping the structural properties of the lumbosacral vertebral endplates. Spine (Phila Pa 1976) 2001;26:889-96.

32. Pal GP, Cosio L, Routal RV. Trajectory architecture of the trabecular bone between the body and the neural arch in human vertebrae. Anat Rec 1988;222:418-25.

33. Trout AT, Kallmes DF, Layton KF, et al. Vertebral endplate fractures: an indicator of the abnormal forces generated in the spine after vertebroplasty. J Bone Miner Res 2006;21:1797-802.

34. Roberts S, McCall IW, Menage J, et al. Does the thickness of the vertebral subchondral bone reflect the composition of the intervertebral disc? Eur Spine J 1997;6:385-9.

35. Edwards WT, Zheng Y, Ferrara LA, et al. Structural features and thickness of the vertebral cortex in the thoracolumbar spine. Spine (Phila Pa 1976)

Cite this article as: Che-Nordin N, Deng M, Griffith JF, Leung JC, Kwok AW, Zhu YQ, So RH, Kwok TC, Leung PC, Wáng YX. Prevalent osteoporotic vertebral fractures more likely involve the upper endplate than the lower endplate and even more so in males. Ann Transl Med 2018;6(22):442. doi: 10.21037/atm.2018.10.61
2001;26:218-25.

36. Grant JP, Oxland TR, Dvorak MF, et al. The effects of bone density and disc degeneration on the structural property distributions in the lower lumbar vertebral endplates. J Orthop Res 2002;20:1115-20.

37. Wáng JQ, Káplár Z, Deng M, et al. Thoracolumbar Intervertebral Disc Area Morphometry in Elderly Chinese Men and Women: Radiographic Quantifications at Baseline and Changes at Year-4 Follow-up. Spine (Phila $\mathrm{Pa}$ 1976) 2018;43:E607-14.

38. Ruiz Santiago F, Tomás Muñoz P, Moya Sánchez E, et al. Classifying thoracolumbar fractures: role of quantitative imaging. Quant Imaging Med Surg 2016;6:772-84.

39. Wáng YX, Wáng JQ, Káplár Z. Increased low back pain prevalence in females than in males after menopause age: evidences based on synthetic literature review. Quant Imaging Med Surg 2016;6:199-206.

40. Lotz JC, Fields AJ, Liebenberg EC. The role of the vertebral end plate in low back pain. Global Spine J 2013;3:153-64.

41. Wáng YX, Wu AM, Ruiz Santiago F, et al. Informed appropriate imaging for low back pain management: A narrative review. J Orthop Translat 2018;15:21-34.

42. Han IH, Chin DK, Kuh SU, et al. Magnetic resonance imaging findings of subsequent fractures after vertebroplasty. Neurosurgery 2009;64:740-4; discussion 744-5.

43. Chan KK, Sartoris DJ, Haghighi P, et al. Cupid's bow contour of the vertebral body: evaluation of pathogenesis with bone densitometry and imaging-histopathologic correlation. Radiology 1997;202:253-6. 
Supplementary

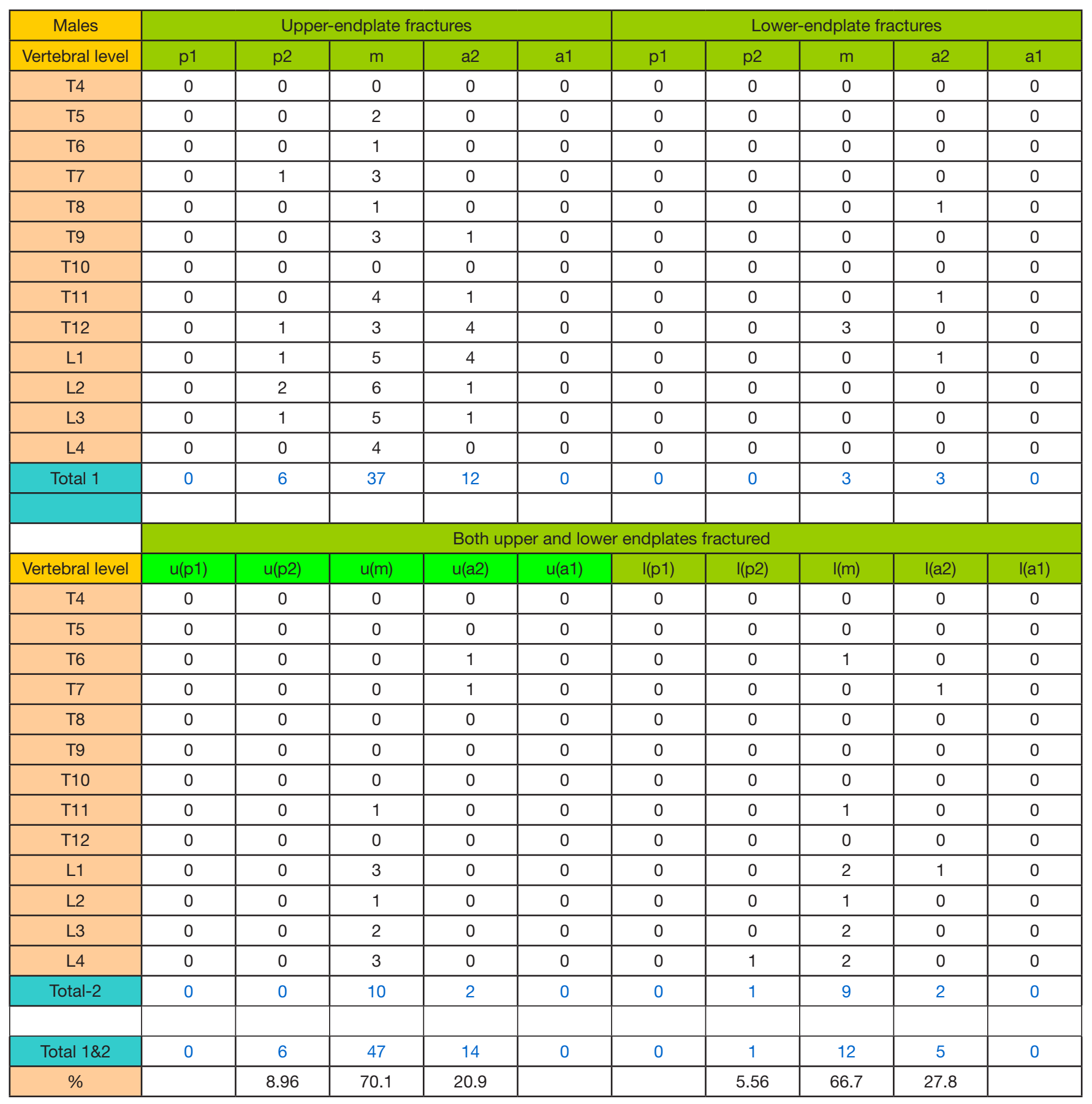

Figure S1 Male subjects data (excluding grade 3 deformities) distribution of osteoporotic endplate fracture in anteroposterior 5-segments of endplate (from anterior to posterior: a1, a2, m, p2, p1). u(p1), segment p-1 of upper endplate; 1(p1), segment p-1 of lower endplate. 


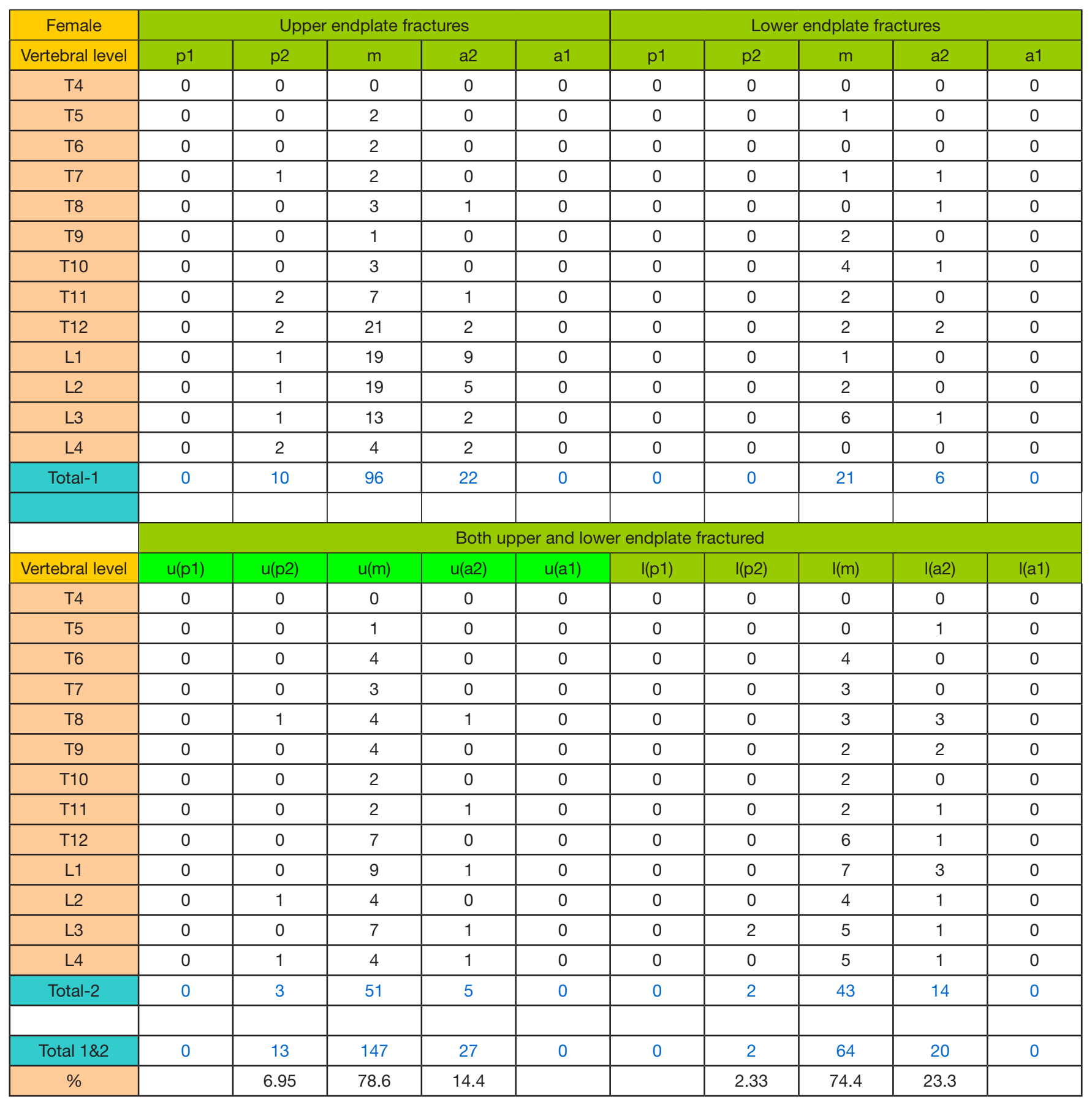

Figure S2 Female subjects data (excluding grade 3 deformities) distribution of osteoporotic endplate fracture in anteroposterior 5 -segments of endplate (from anterior to posterior: a1, a2, m, p2, p1). u(p1), segment p-1 of upper endplate; 1(p1), segment p-1 of lower endplate. 